\title{
UNIQUENESS OF UNBOUNDED LOEB MEASURE USING CHOQUET'S THEOREM
}

\author{
BOŠKO ŽIVALJEVIĆ
}

(Communicated by Andreas R. Blass)

\begin{abstract}
Uniqueness of the Carathéodory extension of the standard part map of an internal unbounded measure $\mu$ defined on an internal algebra $\mathscr{A}$ of subsets of an internal set $\Omega$ has been proved by Henson using the notion of a countably determined set. Here we show how Choquet's capacitability theorem can be used in the proof of the same result.
\end{abstract}

Uniqueness of the Carathéodory extension of an internal measure $\mu$ defined on an internal algebra $\mathscr{A}$ of subsets of an internal set $\Omega$ is proved by Henson in [H2]. In that paper the author introduces the notion of a countably determined set and proves a few lemmas about those sets, which, in turn, enable him to prove the uniqueness of the extension of the measure $\mu$ over the $\sigma$-algebra generated by internal algebra $\mathscr{A}$. In this paper we show how Choquet's capacitability theorem can be used when countably determined sets are replaced by Souslin sets. Although the class of countably determined sets is much wider than the class of Souslin sets (the class of countably determined sets contains the class of projective sets of finite rank), which makes the results obtained by Henson more general, we believe that the proof we present here gives an interesting example of the more extensive use of Choquet's theorem in Nonstandard Analysis (see [HR, R, Ž $1, \check{Z} 3]$ ).

An internal measure space $(\Omega, \mathscr{A}, \mu)$ consists of an internal set $\Omega$, an internal algebra $\mathscr{A}$ of internal subsets of $\Omega$, and an internal *-finitely additive measure $\mu$ defined on elements of algebra $\mathscr{A}$. If $\mu(\Omega)$ is a finite element of ${ }^{*} R$ we say that $\mu$ is bounded; otherwise the measure $\mu$ is unbounded. As was noticed by Loeb [Lo], if the nonstandard universe is $\omega_{1}$-saturated the measure st $\circ \mu$ (st being the standard part map) satisfies Carathéodory's condition for extensions of finitely additive measures and, thus, can be extended to a standard $\sigma$-additive measure $L(\mu)$ defined on the $\sigma$-algebra $\sigma(\mathscr{A})$ generated by the algebra $\mathscr{A}$. Sets in the $\sigma$-algebra $\sigma(\mathscr{A})$ are also called Borel over $\mathscr{A}$.

By $\omega$ we denote the set of integers, by $\omega^{<\omega}$ the set of all finite sequences of integers, and by $\omega^{\omega}$ the set of all infinite sequences of integers. Given a finite sequence $s$ of integers the length of $s$ is defined as the number of elements of

Received by the editors July 19, 1989 and, in revised form, March 7, 1991.

1991 Mathematics Subject Classification. Primary 03H05; Secondary 28A05, 28 A12.

The research supported by the National Science Foundation and the Research Board of the University of Illinois. 
the sequence. By $\omega^{n}$ we denote the set of all sequences of integers of length $n$. Note that 0 is included in the set $\omega$ so that a typical element of the set $\omega^{n}$ is a sequence $\left(p_{0}, \ldots, p_{n-1}\right)$. Given an element $s \in \omega^{<\omega} \cup \omega^{\omega}$ and an integer $n$ we define $s \mid n$ to be the restriction of $s$ to its $n$ first places, i.e., $s \mid n=(s(0), \ldots, s(n-1))$ if $n \neq 0$ and $s \mid n=\varnothing$ otherwise. Here we suppose that the length of $s$ is $\geq n$; if the length of $s$ is less than $n$ we put $s \mid n=s$.

A subset of $\Omega$ is called Souslin if there exists a system $A_{s}\left(s \in \omega^{<\omega}\right)$ of internal subsets of $\Omega$ such that

$$
S=\bigcup_{\xi \in \omega^{\omega}} \bigcap_{n \in \omega} A_{\xi \mid n} .
$$

If every $A_{s}$ belongs to an algebra $\mathscr{A}$ of internal subsets of the set $\Omega$ then $S$ is said to be Souslin over $\mathscr{A}$. The above representation of the set $S$ is regular if $s \supset t$ implies $A_{s} \subseteq A_{t}$. The system of sets $A_{s}\left(s \in \omega^{<\omega}\right)$ is in that case called regular. It is well known that every Borel set is Souslin (see for example [JR]) and that a set is Souslin if and only if it can be obtained as a projection of a Borel set in the product $\Omega \times Y$ for some auxiliary internal set $Y$ (see [H1]). For an extensive treatment of the Descriptive Set Theory of Hyperfinite Sets the reader is referred to [KKML].

The approach to Choquet's theorem that we present here is a little bit different from (but also equivalent to) the original approach due to Choquet [Ch] and it can be found in [BS]. Instead of dealing with a certain sort of set functions (capacities), we rather work with pairs of properties, which is called a capacitance pair property. The definition is adapted to the case of internal sets.

Definition 1. Let $\mathscr{A}$ be an algebra of internal set of an internal set $\Omega$. A pair $(\varphi, \psi)$ of properties of subsets of $\Omega$ is called a capacitance pair property over $\mathscr{A}$ if the following three conditions are satisfied:

(i) If $A_{n} \quad(n \in \omega)$ is an ascending sequence of arbitrary subsets of $\Omega$ with $\bigcup_{n \in \omega} A_{n}$ possessing the property $\varphi$ then $A_{m}$ possesses the property $\varphi$ for some $m \in \omega$.

(ii) If a set $A$ possesses the property $\varphi$ and $A \subseteq B$ then $B$ possesses the property $\varphi$ also.

(iii) If $F_{n} \quad(n \in \omega)$ is a decreasing sequence of members of $\mathscr{A}$ processing the property $\varphi$ then $\bigcap_{n \in \omega} F_{n}$ possesses the property $\psi$.

Now we state Choquet's capacitability theorem in a way that was presented in [BS]. We restrict our attention only to internal sets, as usual.

Proposition 2 [C, BS]. Let $\mathscr{A}$ be an algebra of internal set of an internal set $\Omega$, and let $(\varphi, \psi)$ be a capacitance pair property over $\mathscr{A}$. Let $S$ be a Souslin set over $\mathscr{A}$ possessing the property $\varphi$. Then, there exists a descending sequence $P_{n}$ $(n \in \omega)$ of elements of $\mathscr{A}$ possessing the property $\varphi$ such that $\bigcap_{n \in \omega} P_{n} \subseteq S$ and such that $\bigcap_{n \in \omega} P_{n}$ possesses the property $\psi$.

We are ready now to prove a result from [H2] (Theorem 1 in [H2]), which when restricted to Borel sets immediately proves the uniqueness of the existence of unbounded Loeb measure over the algebra of Borel sets.

Proposition 3 [H2]. Let $(\Omega, \mathscr{A}, \mu)$ be an unbounded internal measure space. Let $S \subseteq \Omega$ be a Souslin set over $\mathscr{A}$. Then, either there exists $A \in \mathscr{A}$ with 
$A \subseteq S$ and $A$ of infinite $\mu$ measure or $S$ can be covered by countably many sets from $\mathscr{A}$ of finite $\mu$ measure.

Proof. Let $\varphi$ be a property of a set $S$ defined as: "S cannot be covered by countably many sets from $\mathscr{A}$ of finite $\mu$ measure," and let $\psi$ be a property of $S$ defined as: "there exists an element $A$ of $\mathscr{A}$ of infinite $\mu$ measure with $A \subseteq S$." We show that a pair $(\varphi, \psi)$ is a capacitance pair property.

Indeed, if a set $M$ possesses the property $\varphi$, that is, if $M$ cannot be covered by countably many internal sets from $\mathscr{A}$ of finite $\mu$ measure, then the same holds for every set $N$ containing $M$. This verifies the property (ii) of Definition 1.

If $\bigcup_{n \in \omega} M_{n}$ is an increasing union of sets such that $\bigcup_{n \in \omega} M_{n}$ is not possible to cover by countably many elements from $\mathscr{A}$ of finite $\mu$ measure, then obviously the same is true for some $M_{k}$. This shows (i) from Definition 1 .

Finally, to verify the property (iii) from Definition 1, let $A_{n} \quad(n \in \omega)$ be a decreasing sequence of sets from $\mathscr{A}$, each of which is not possible to cover by countably many elements of $\mathscr{A}$ of finite $\mu$ measure. Then, in particular, the $\mu$ measure of each $A_{n}$ is infinite, and by $\omega_{1}$-saturation there exists an element $B$ of $\mathscr{A}$ a subset of $\bigcap_{n \in \omega} A_{n}$ and of infinite $\mu$ measure. That means that the intersection $\bigcap_{n \in \omega} A_{n}$ possesses the property $\psi$.

We have proved that the pair $(\varphi, \psi)$ is a capacitance pair property.

So, if $S$ cannot be covered by countably many elements of $\mathscr{A}$ of finite $\mu$ measure, i.e., if $S$ possess the property $\varphi$, then by Proposition 2 (Choquet's capacitability theorem) there exists a set $P \subseteq S$ possessing the property $\psi$. That is, $P$ and thus $S$ contains an element $A$ of $\mathscr{A}$ of infinite $\mu$ measure.

Remark. Corollary 2 to Theorem 1 in [H2] states the following: if $S \in \sigma(\mathscr{A})$ then either there exists $A \in \mathscr{A}$ such that $S \subseteq A$ and $\mu(A)$ finite or for each $n \in \omega$ there exists $A_{n} \in \mathscr{A}$ such that $A_{n} \subseteq S$ and $\mu\left(A_{n}\right) \geq n$. That can also be proved using the capacitability argument as follows. Let for $n \in \omega, \varphi_{n}$ be the property of a set $M$ defined as: "there does not exist $A \in \mathscr{A}$ such that $M \subseteq A$ and $\mu(A) \leq n$," and let $\psi_{n}$ be defined as: "there exists $A \in \mathscr{A}$ such that $A \subseteq M, \mu(A) \geq n$." Then the pair $\left(\varphi_{n}, \psi_{n}\right)$ is a capacitance pair property. (The property (ii) of a capacitance pair property is trivial, while (i) and (iii) follow from $\omega_{1}$-saturation.)

Now let $S$ be a Souslin over $\mathscr{A}$ set. If there is no set $A \in \mathscr{A}$ such that $S \subseteq A$ and $\mu(A)$ is finite, then $S$ possesses the property $\varphi_{n}$ for every $n$. By Choquet's theorem for every $n \in \omega$ there exist a subset of $S$ possessing the property $\psi_{n}$.

Let $(\Omega, \mathscr{A}, \mu)$ be, as usual, an internal measure space. Let $\Delta$ be a set of positive infinitesimals satisfying

$$
\begin{gathered}
0 \leq \eta<\delta \in \Delta \text { implies } \eta \in \Delta, \\
\eta, \delta \in \Delta \text { implies } \eta+\delta \in \Delta
\end{gathered}
$$

A set $M$ is said to be $\Delta$-null if for every $p>\Delta$ there exists an $A \in \mathscr{A}$ such that $M \subseteq A$ and $\mu(A)<p$. Here $p>\Delta$ means that $p>q$ for every $q \in \Delta$.

Next we prove a version of Theorem 2 from [H2]. The theorem states that a Borel set $S$ over $\mathscr{A}$ is $\Delta$-null if and only if for every $A \subseteq S, A \in \mathscr{A}$ 
we have $\mu(A) \in \Delta$. Henson proves in the same theorem that if $\Delta$ is nonArchimedean (meaning that for every $p>\Delta$ there exists $q>\Delta$ such that $p / q$ is infinite) then the result holds true for any element of the complete Boolean algebra generated by some countable subfamily $\mathscr{C}$ of $\mathscr{A}$ (these sets are called countably determined sets).

Proposition 4 [H2]. Let $S$ be a Souslin set over $\mathscr{A}$. Then $S$ is $\Delta$-null if and only if for every $A \subseteq S, A \in \mathscr{A}$ we have $\mu(A) \in \Delta$.

Proof. If $S$ is $\Delta$-null then the above condition is obviously satisfied. Conversely, suppose that $\mu(A) \in \Delta$ for every $A \in \mathscr{A}, A \subseteq S$. Let $p>\Delta$. Let $\varphi_{p}$ be a property of a set $M$ defined as: "there does not exist an element $A \in \mathscr{A}$ with $A \supseteq M$ and $\mu(A) \leq p$." Let $\psi_{p}$ be a property of $M$ defined as: "there exists an $A \in \mathscr{A}, A \subseteq S$ with $\mu(A) \geq p$." The pair $\left(\varphi_{p}, \psi_{p}\right)$ is a capacitance pair property. Indeed, if $L$ has the property $\varphi_{p}$ then the same is true for every $M \supseteq S$. Also, if $\bigcup_{n \in \omega} L_{n}$ is an increasing union of sets such that $\bigcup_{n \in \omega} L_{n}$ possesses the property $\varphi_{p}$ then some $L_{n}$ must also possess the property $\varphi_{p}$. Otherwise, for every $n \in \omega$ there would exist an element $A_{n} \in \mathscr{A}$ with $A_{n} \supseteq L_{n}$ and $\mu\left(A_{n}\right) \leq p$. Then, using $\omega_{1}$-saturation, we could produce an element $A \in \mathscr{A}$ with $A \supseteq \bigcup_{n \in \omega} A_{n}$ and $\mu(A) \leq p$. Having in mind that $\bigcup_{n \in \omega} L_{n} \subseteq \bigcup_{n \in \omega} A_{n}$, we reach a contradiction.

Finally, if $A_{n} \quad(n \in \omega)$ is an increasing sequence of elements of $\mathscr{A}$ possessing the property $\varphi_{p}$ then, in particular, we have $\mu\left(A_{n}\right)>p$. By $\omega_{1}$-saturation there exists an $A \in \mathscr{A}$ with $A \subseteq \bigcap_{n \in \omega} A_{n}$ and $\mu(A) \geq p$. That means that the $\bigcap_{n \in \omega} A_{n}$ possesses the property $\psi_{p}$.

We have proved that the pair $\left(\varphi_{p}, \psi_{p}\right)$ is a capacitance pair property.

Now the proof is clear. Suppose that $S$ is not $\Delta$-null and that the conditions of the proposition are satisfied. Then $S$ possesses the property $\varphi_{p}$ for some $p>\Delta$. By Choquet's capacitability theorem $S$ possesses the property $\psi_{p}$, which is a contradiction.

\section{REFERENCES}

[BS] D. W. Bressler and M. Sion, The current theory of analytic sets, Canad. J. Math. 16 (1964), 207-230.

[C] G. Choquet, Theory of capacities, Ann. Inst. Fourier (Grenoble) 5 (1955), 131-295.

[H1] C. W. Henson, Analytic sets, Baire sets and the standard part map, Canad. J. Math. 31 (1979), 663-672.

[H2] _ Unbounded Loeb measures, Proc. Amer. Math. Soc. 74 (1979), 143-150.

[HR] C. W. Henson and D. Ross, Analytic mappings on hyperfinite sets (to appear).

[HuLo] A. E. Hurd and P. A. Loeb, An introduction to nonstandard real analysis, Academic Press, New York, 1985.

[JR] J. E. Jane and C. A. Rogers, K-analytic sets, Analytic Sets (C. A. Rogers, ed.), Academic Press, New York, 1980.

[KKML] H. J. Keisler, K. Kunen, A. Miller, and S. Leth, Descriptive set theory over a hyperfinite set, J. Symbolic Logic 54 (1989), 1167-1180.

[Lo] P. A. Loeb, Conversion from nonstandard to standard measure spaces and application in probability theory, Trans. Amer. Math. Soc. 211 (1975), 113-122.

[R] D. Ross, Lifting theorems in nonstandard measure theory, Proc. Amer. Math. Soc. 109 (1990), 809-822. 
[Ž1] B. Živaljević, Every Borel function is monotone Borel, Ann. Pure Appl. Logic 54 (1991), 87-99.

[Ž2] _ Some results about Borel sets in descriptive set theory of hyperfinite sets, J. Symbolic Logic 55 (1990), 604-614.

[Ž3] _ $\quad U$-meager sets when the cofinality and the coinitiality of $U$ are uncountable, $\mathrm{J}$. Symbolic Logic 56 (1991), 906-914.

Department of Mathematics, University of Illinois, Urbana, Illinois 61801

Current address: Department of Computer Science, Michigan State University, A713 Wells Hall, East Lansing, Michigan 48824 